\title{
Utilization of Moringa oleifera leaves powder as a functional food ingredient in traditional food product
}

\section{S.M. Shinde and K.P. Babar}

Received : 10.04.2018; Accepted : 13.04 .2018

See end of the Paper for authors' affiliation

Correspondence to :

\section{S.M. Shinde}

Department of Agricultural Engineering, Maharashtra Institute of Technology, Aurangabad (M.S.) India Email : swapnashinde 43@ gmail.com
- Abstract : Moringa oleifera is a multipurpose and nutritious vegetable tree with a variety of potential uses and its leaf is most nutritious. Dehydration is carried out by three methods i.e. tray drying, sun drying shade drying. Drying of Moringa leaf powder not only increases the micronutrients but also increases the shelf life of its powder. The nutritional potential and antioxidant component present in Moring a make it suitable for preparation of various traditional products. So Moringa powder is fortified as functional food ingredient inparatha. The main ingredients in Paratha are wheat flour and refined wheat flour is deficient in protein, vitamins and minerals. Use of Moringa leaves powder in paratha not only increases the nutritional value but also help to resolve problem of malnutrition. It has many health benefits like antioxidant, it prevent skin diseases, diabetes, cancer, cold and flu, proper digestion, anti-tumour, anti-inflammatory, cholesterol lowering,anti-bacterial and anti-fungal properties etc. The objectives of this review to study the nutritional or proximate composition of Moringa paratha and evaluate its acceptability through sensory evaluation tests.

- Key words : Moringa oleifera leaves, Health benefits, Paratha

- How to cite this paper : Shinde, S.M. and Babar, K.P. (2018). Utilization of Moringa oleifera leaves powder as a functional food ingredient in traditional food product. Internat. J. Agric. Engg., 11(Sp. Issue) : 102-104, DOI: 10.15740/HAS/IJAE/11.Sp. Issue/102-104. 